\title{
Mutual event observations of solar system objects by SRC on Mars Express ${ }^{\star}$
}

\section{Analysis and release of observations}

\author{
R. Ziese ${ }^{1}$ and K. Willner ${ }^{2}$ \\ ${ }^{1}$ Technische Universität Berlin, Institute of Geodesy and Geoinformation Science, Str. des 17. Juni 135, 10628 Berlin, Germany \\ e-mail: ziese@tu-berlin.de \\ ${ }^{2}$ German Aerospace Center (DLR), Institute of Planetary Research, Rutherfordstr. 2, 12489 Berlin, Germany \\ e-mail: konrad.willner@dlr.de
}

Received 25 July 2017 / Accepted 21 January 2018

\begin{abstract}
Context. Both Martian moons, Phobos and Deimos, have been observed during several imaging campaigns by the Super Resolution Channel (SRC) on the Mars Express probe. Several tens of images are obtained during mutual event observations - when the Martian moons are both observed or together with another solar system body. These observations provide new opportunities to determine the bodies' positions in their orbits.

Aims. A method was sought to automate the observation of the positions of the imaged bodies. Within one image sequence a similarly accurate localization of the objects in all images should be possible.

Methods. Shape models of Phobos and Deimos are applied to simulate the appearance of the bodies in the images. Matching the illuminated simulation against the observation provides a reliable determination of the bodies' location within the image. To enhance the matching confidence several corrections need to be applied to the simulation to closely reconstruct the observation.

Results. A list of 884 relative positions between the different objects is provided through the Centre de Données astronomiques de Strasbourg (CDS).
\end{abstract}

Key words. astrometry - ephemerides - planets and satellites: individual: Phobos - planets and satellites: individual: Deimos

\section{Introduction}

First observed in 1877 by Asaph Hall, an astronomer of the US Naval Observatory in Washington, the two natural Martian satellites, Phobos and Deimos, can only be observed from Earth during Mars oppositions, that is, every two years (Morley 1989). The bright appearance of Mars combined with the moons' relatively small sizes, Phobos having approximately $27 \times 22 \times 18 \mathrm{~km}$ (Willner et al. 2014) diameter and Deimos measuring $16 \times 12 \times 10 \mathrm{~km}$ in diameter, as well as their low orbits, $6000 \mathrm{~km}$ and $20000 \mathrm{~km}$ above the Martian surface, make Earth-based observations difficult.

In the past Phobos' and Deimos' positions in space were observed separately by several spacecraft during their approach to Mars and at times also during flyby or far distance observations, for example, by Mariner 9 (Duxbury \& Callahan 1989), Viking (Duxbury \& Callahan 1988), Phobos 2 (Kolyuka et al. 1991), MGS (Bills et al. 2005; Jacobson 2010, and references therein), MRO (Jacobson 2010; Jacobson \& Lainey 2014) and Mars Express (Oberst et al. 2006; Willner et al. 2008; Pasewaldt et al. 2012, 2015).

Here we focus on observations by the European Mars Express (MEX) probe, which has been in orbit about Mars since

\footnotetext{
* Tables A.1-A.4 are only available at the CDS via anonymous ftp to cdsarc.u-strasbg.fr $(130.79 .128 .5)$ or via http://cdsarc.u-strasbg.fr/viz-bin/qcat?]/A+A/614/A15
}

2003 and carries the High Resolution Stereo Camera (HRSC) designed for multi-spectral mapping of the Martian surface (Jaumann et al. 2007). This camera also features the Super Resolution Channel (SRC), a $1 \mathrm{~K}$ by $1 \mathrm{~K}$ framing camera, which provides high-resolution details within HRSC scenes, thus enabling studies of small-scale geologic features (Oberst et al. 2008). In contrast to other missions, Mars Express is in a highly elliptical nearly polar orbit around Mars. The periapsis height of the MEX orbit is optimized for HRSC observations of Mars to ensure surface resolutions in the range of 10 to $15 \mathrm{~m} \mathrm{pixel}{ }^{-1}$ and is thus at a height of $250 \mathrm{~km}$ above the Martian surface (Jaumann et al. 2007) with a latitudinal precession to assure global coverage. As a consequence, the apoapsis of MEX's orbit is at a distance of about $11000 \mathrm{~km}$ to the Martian surface and thus beyond the orbit of Phobos. The nearly equatorial orbits of Phobos and Deimos allow observations of the moons by MEX instruments from distances of less than $5000 \mathrm{~km}$ and $12000 \mathrm{~km}$, respectively, at an interval of five to six months.

Several different techniques have been applied to gather astrometric information from SRC images (see Oberst et al. 2006; Willner et al. 2008; Pasewaldt et al. 2012, 2015). In contrast to the method that is described below, Willner et al. (2008) and Pasewaldt et al. $(2012,2015)$ used stars in the background to control the camera pointing for astrometric observations. According to Willner et al. (2008) pointing errors of MEX mainly relate to attitude uncertainties about the $X$ and $Y$ axes of the camera leading to translations of the observed 
bodies' location in the image. Compared to these translational errors, rotations about the boresight axis can be neglected (Willner 2009).

The HRSC planning team has identified occasions where both moons or one of the satellites together with another planetary object, for example, a planet, moon, star or star field, can be observed. This kind of observation is referred to as mutual event. Usually during such occasions a sequence of several tens of images is obtained. These observations provide a unique opportunity to derive information on the relative position of the Martian moons with respect to another distant but well-known celestial object. However, no analysis of these data has been carried out to date.

The advantage of mutual event observations over observations of only one Martian moon is that the absolute camera pointing is irrelevant as long as two objects are visible and the relative angular separation between the two bodies can be determined. This leads to accurate position information of Phobos or Deimos with respect to one of the other, above-mentioned celestial bodies. Furthermore, mutual event observations can be obtained more frequently than direct observations during flybys. Distance is considered less critical in theses cases due to the independency from the spacecraft pointing and thus observations from a wide range of MEX orbit positions are possible. Both features make this kind of observation an ideal input to orbit modeling algorithms.

Improved ephemerides for Phobos and Deimos have some immediate benefits for navigational purposes. However, besides from being an indispensable prerequisite for spacecraft mission design, orbit planning and optical navigation, accurate ephemerides also serve scientific objectives.

The ephemerides are derived by numerically integrating the satellite orbits while simultaneously fitting these to the astrometric observations (Lainey et al. 2007; Jacobson 2010; Jacobson \& Lainey 2014). Dynamical model parameter values are also determined during the least-squares adjustment, including the amplitude of Phobos' libration in longitude and Phobos' secular acceleration (Jacobson 2010).

Variations in the rotational motion (i.e., librations) impose constraints on the mass distribution inside Phobos on a finer scale (Rambaux et al. 2012). Combined with a precise knowledge of the secular drift of the periapsis of Phobos' orbit, they allow conclusions on the satellites' second-order gravity field coefficents (Borderies \& Yoder 1990; Jacobson \& Lainey 2014). Variations in the orbital motion of the Martian moons are also a means of studying Mars itself. Temporal accelerations can be used to investigate the Martian $J_{2}$ gravity coefficient, in particular its seasonal changes. Furthermore, tidal accelerations allow the determination of the rate of tidal dissipation (Lainey et al. 2007; Bills et al. 2005) and yield constraints on the mechanical properties of the Martian interior, for example, its viscoelastic behavior.

GM estimates for Phobos have been derived, among others, by Christensen et al. (1977), Konopliv et al. (2006) and Jacobson (2010), but most recently from MEX tracking data during close Phobos flybys (Pätzold et al. 2014b, a). For a more comprehensive list of studies related to the GM of Phobos and Deimos, see Jacobson (2010) and Pätzold et al. (2014b).

However, to reduce these data, accurate orbit modeling for both the spacecraft and Phobos is required.

\section{Observations}

Here, observations showing either both Martian moons within one SRC image or one of the moons with Jupiter or Saturn in
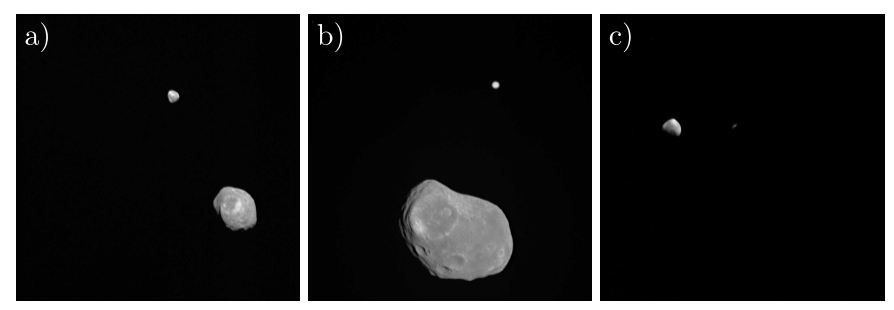

Fig. 1. Measurements showing $a$ ) Phobos and Deimos (HD090_0032), b) Phobos and Jupiter (HC151_0085) and c) Deimos together with Saturn (HC921_0030).

the background are analyzed (Fig. 1). Next to these mutual event observations, a wealth of data exist imaging Phobos or Deimos and a star or star field. A few observations containing the Earth's moon and the Galilean moons exist. These bodies are, due to their size and distance, comparable to point-like light sources and appear similar to star records in SRC images. As this kind of observation requires a different measurement approach, their analysis will be discussed in a separate publication.

In total there are 1024 images showing two planetary objects (cf. Table 1). If either Phobos or Deimos is imaged together with the Jovian system or Saturn, then the Martian moon is designated as the primary object and the other planetary body as the secondary object. If Phobos and Deimos are imaged, then Phobos is considered as the primary object.

During mutual event observations the apparent viewing geometry between camera and primary object (e.g., Phobos or Deimos) changes only on a minimal scale. Hence, initially it was assumed that the perspective between observer and primary object as well as secondary object does not change significantly throughout one observation series. Under this assumption, techniques to detect the observed features automatically can be applied assuring consistency in the quality of observations throughout one image sequence.

However, a detailed analysis revealed that the observation geometry changes throughout long observation series or simply due to an unfortunate imaging geometry, for example, viewing Phobos from an angle from which small changes of the observers' position changes the image significantly. In these cases a segmentation of the image series was applied; see Sect. 3.3.

Other than the secondary object, which is usually completely visible in all images, the primary object enters and leaves the field of view (FOV) throughout one image series. Images showing more than approximately $15 \%$ of the primary object are evaluated (see Sect. 3.2), images showing a lesser portion of the primary object are excluded from further analysis.

There are three series, in which some images show an occultation of the secondary object by the primary object: In the MEX orbits 12921 and 13228, Saturn is occulted by Deimos and Phobos, respectively; in orbit 9463 , Jupiter is partially occulted by Phobos. The corresponding images as well as images in which the secondary and the primary object are too close to each other to be recognized as separate objects by automated procedures are excluded from further evaluation.

Table 1 provides an overview of the mutual event observations until January 2017. The first three columns list the MEX orbit number for each series, the primary object, and the secondary object, respectively. The fourth column displays the number of all images of the series showing both the primary and secondary object. This number also includes images that were, for any of the above-mentioned reasons, deemed as unsuitable 
Table 1. Mutual event observations until January 2017.

\begin{tabular}{cllcc}
\hline \hline $\begin{array}{c}\text { MEX orbit } \\
\text { number }\end{array}$ & $\begin{array}{l}\text { Primary object } \\
\text { observed }\end{array}$ & $\begin{array}{l}\text { Secondary object } \\
\text { observed }\end{array}$ & $\begin{array}{c}\text { No. mutual event } \\
\text { images }\end{array}$ & $\begin{array}{c}\text { No. analyzed } \\
\text { images }\end{array}$ \\
\hline 7492 & Phobos & Deimos & 54 & 35 \\
9463 & Phobos & Jupiter & 72 & $62^{a}$ \\
9700 & Phobos & Deimos & 88 & 76 \\
12146 & Phobos & Deimos & 32 & 27 \\
12151 & Phobos & Jupiter & 107 & 84 \\
12921 & Deimos & Saturn & 61 & $53^{b}$ \\
12963 & Phobos & Saturn & 91 & 87 \\
13090 & Phobos & Deimos & 61 & 58 \\
13228 & Phobos & Saturn & 99 & $79^{c}$ \\
13320 & Phobos & Saturn & 99 & 99 \\
15812 & Deimos & Saturn & 58 & 56 \\
15880 & Phobos & Deimos & 11 & 0 \\
15898 & Deimos & Saturn & 76 & 73 \\
16205 & Phobos & Saturn & 11 & 9 \\
16346 & Phobos & Saturn & 25 & 21 \\
16379 & Phobos & Deimos & 41 & 38 \\
16569 & Phobos & Deimos & 38 & 27 \\
\hline
\end{tabular}

Notes. ${ }^{(a)}$ Phobos partially occults Jupiter; ${ }^{(b)}$ Deimos occults Saturn; ${ }^{(c)}$ Phobos occults Saturn.

for evaluation. The fifth column, however, states the number of images that were actually evaluated.

\section{Methods}

The actual measurement is the determination of the image coordinates of a body's center of figure (COF). Therefor, the observation geometry is simulated using the NAIF SPICE toolkit (cspice, version N0065) applying (a) shape models in the case of Phobos (Willner et al. 2014) and Deimos (Thomas et al. 2000) or ellipsoid models for planets with additional ring models in the case of Saturn (Acton 2013) ${ }^{1}$, (b) an Akimov disk function light model in the case of a rocky object (Shkuratov et al. 1999; Longobardo et al. 2014) and (c) a function describing the limb darkening effect (Milne 1921) for the gas giants Jupiter and Saturn to achieve a most realistic prediction of the scene. The simulation of the objects is used to detect the objects in one image of each series. A cut-out of this image containing only the detected object is created, and used as a template for the matching process in all other images of this specific observation series. Besides the automatic detection of an object in the image, the simulated template has another advantage. As the shape model is used to simulate the observation, the COF location is known for the simulation and its location in the image is determined once a good match between observation and simulation is achieved.

\subsection{Simulation}

For each mutual event, one image is chosen as a reference. One simulation for each of the planetary objects is computed based on (a) the reference image acquisition time; (b) ephemeris data for MEX, the SRC's nominal pointing, and the camera parameters obtained after an in-flight calibration (see Oberst et al. 2008 and

\footnotetext{
1 See also the SPICE Geometry Finder Required Reading document, https://naif.jpl.nasa.gov/pub/naif/toolkit_docs/ C/req/gf.html\#Approximate\%20times\%20of\%20Cassini\% 20Saturn\%20ring\%20occultations
}

Duxbury et al. 2011); and (c) ephemeris data for the planetary objects as well as models for their rotation and shape. Jupiter, Saturn's body and Earth are treated as ellipsoids. Saturn's rings are modeled separately, as is described at the end of this section. To simulate the appearance of Phobos and Deimos in the images, shape models derived by Willner et al. (2014) and Thomas et al. (2000), respectively, are used. The shape models used to simulate the observed object provide the COF of the respective body. However, ephemerides contain the position of the center of mass (COM) of an object. For Phobos and Deimos no offsets between the $\mathrm{COF}$ and $\mathrm{COM}$ are known, or are, if existent, believed to be very small. For this analysis it is assumed that both centers coincide.

In the case of Phobos and Deimos, the solar incidence angle $i$, emission angle $e$, and phase angle $p$ are computed for each point on the surface. Moreover, for each surface point, we determined whether it was shadowed by other parts of the moon; see Fig. 2. Using these data, the body's reflectance properties are modeled by the (parameterless) Akimov disk function (Shkuratov et al. 1999; Longobardo et al. 2014), given by

$\mathrm{f}(i, e, p)= \begin{cases}\cos \frac{p}{2} \times \cos \left(\frac{\pi}{\pi-p} \times\left(\gamma-\frac{p}{2}\right)\right) \times \frac{(\cos \beta)^{\frac{p}{\pi-p}}}{\cos \gamma}, & \text { if } i<=\frac{\pi}{2} \\ 0, & \text { else }\end{cases}$

where

$$
\begin{aligned}
\gamma(i, e, p) & :=\arctan \left(\frac{\cos i-\cos e \times \cos p}{\cos e \times \sin p}\right), \\
\beta(e, \gamma) & :=\arccos \left(\frac{\cos e}{\cos \gamma}\right),
\end{aligned}
$$

are the photometric longitude and the photometric latitude, respectively.

For the gas giants Jupiter and Saturn (without its rings) however, Akimov's disk function does not provide an adequate simulation. Similar to the Sun they show a darkening of the limb, 

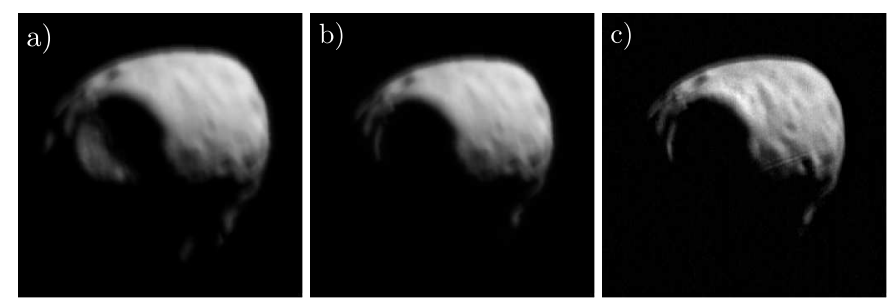

Fig. 2. Image HC563_0008, Phobos: a) Simulation without selfshadowing, rescaled to fit the measurement, $b$ ) Simulation including self-shadowing, rescaled to fit the measurement, $c$ ) Measurement.
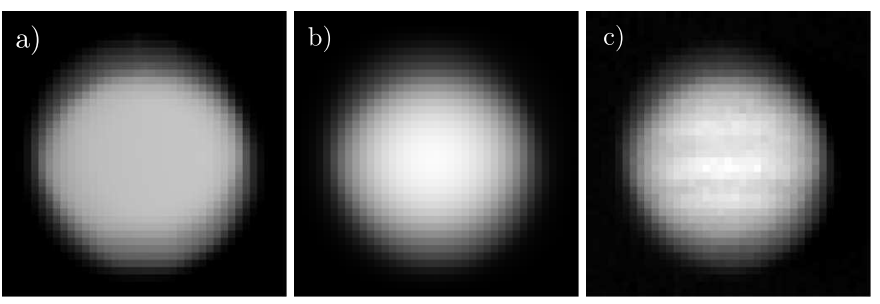

Fig. 3. Image HC151_0085, Jupiter: a) Simulation without the limb darkening effect, rescaled to fit the measurement, $b$ ) Simulation including the limb darkening effect, rescaled to fit the measurement, c) Measurement.
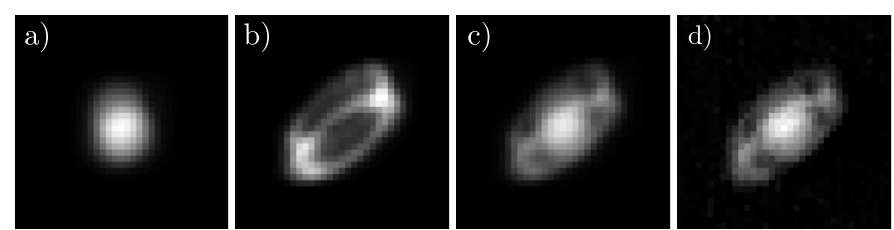

Fig. 4. Image HC921_0030: Simulations of $a$ ) Saturn itself, $b$ ) Saturn's rings, $c$ ) Saturn together with its rings, $d$ ) Measurement.

which is modeled by assigning the cosine of the corresponding emission angle to each pixel; see Fig. 3.

In a first step Saturn and its rings are modeled separately. Saturn itself is simulated as an ellipsoid, taking into account the limb darkening effect as described above (see Fig. 4a). Adopting an idea from Acton $(2013)^{1}$, the Saturnian rings are modeled using two auxiliary ellipsoidal objects having the same COM and the same ephemeris data as Saturn, but with a very small polar radius of only $0.01 \mathrm{~km}$ and different equatorial radii. One auxiliary object has an equatorial radius of $125000 \mathrm{~km}$ and represents the outer boundary of Saturn's ring system. The other has an equatorial radius of $85000 \mathrm{~km}$ and represents the inner boundary. For both these auxiliary ellipsoids the reflectance properties are modeled by the Akimov disk function as outlined above. A simulation for the rings is then obtained by taking the difference of both auxiliary simulations (see Fig. 4b). At last, the total simulation of Saturn together with its rings is computed by combining the simulation of Saturn itself and the simulation of the rings (Fig. 4c).

To achieve a better agreement with the observation, the simulation is convolved with a point spread function (PSF) describing the image distortion of the SRC. Here a function comprised of a $9 \times 9$ grid of pixels - a subset of the PSF derived by Duxbury et al. (2011) - was applied. At last, cut outs from the total simulated images provide simulations for both objects, for which the coordinates of the objects' COFs with respect to the upper left corner are known.
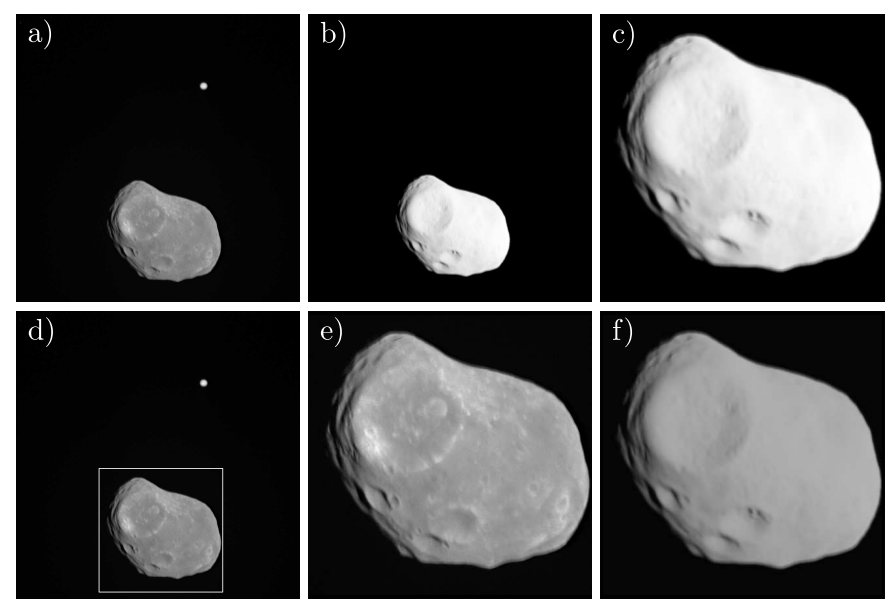

Fig. 5. Panel $a$ : Measured image HC151_0085, panel $b$ : Simulation of Phobos, panel c: Template for Phobos (cut-out of simulation), panel $d$ : Template identified in measured image, panel $e$ : New template for Phobos, panel $f$ : Rescaled template for Phobos (for least-squares fit).

\subsection{Automated matching}

SRC images contain a number of so-called dead pixels, that is, pixels that are unresponsive to incoming light and appear dark regardless of the image displayed. They can be the result of manufacturing anomalies or can occur later on if the pixel is permanently cut-off from its power supply. In the case of the SRC most of the dead pixels result from a powerful particle bombardment of the CCD caused by strong solar flares during MEX's cruise to Mars (Oberst et al. 2008). The dead pixels always appear at the same positions in the image while the observed bodies move through the FOV and thus change their positions from image to image. Strong gray value variations due to the dead pixel distribution may occur between the matching template and the current observation. This impedes an accurate matching. Hence, dead pixels values are replaced by the median of the intensities of the surrounding pixels during a preprocessing step.

In a first step each object is located in the reference image based on the simulated template and using a normal cross correlation (NCC) matching routine (cf. Fig. 5 plates (a to c)). This determines the $\mathrm{COF}$ location of the respective object in the reference image at pixel-level accuracy, as the NCC matching leads to results accurate to one pixel. A rectangular section of the reference image containing the real observation of the body is defined as a new template to be applied for matching all other images of the sequence (Fig. 5 plate ( $\mathrm{d}$ and f)).

To improve the matching result to sub-pixel accuracy level, least-squares matching is applied. However, it turned out to be necessary to rescale the simulated template brightness to better agree with the real observation. This is achieved by leveling the mean intensity and intensity standard deviation between simulated template (Fig. 5c) and template (Fig. 5e) into agreement. This results in a simulated matching template that differs in shading almost solely due to the albedo differences on the surface of Phobos (Fig. 5f). The least squares fit not only allows translations but also rotations of the template and thus takes care of a possible rotation of the spacecraft about the boresight axis of the camera. During the matching, the simulated template is not rescaled in size since comparisons of simulations and measurements have shown that a rescaling of the simulated objects' size is unnecessary. Moreover, Willner et al. (2008) has shown that a possible scaling factor is very close to 1 and is thus negligible. 
Finally, for each of the simulated objects the corresponding section from the reference image is used as a template for the matching process in all other images of this specific observation series.

During each fit the accordance of template and measurement is assessed by the root mean square deviation of the differences between the corresponding intensities. If observation and template are represented by $n$-dimensional vectors $\boldsymbol{A}$ and $\boldsymbol{B}$, respectively, then their root mean square deviation $F$ is defined by

$$
F(\boldsymbol{A}, \boldsymbol{B})=\frac{\|\boldsymbol{A}-\boldsymbol{B}\|_{L^{2}}}{\|\boldsymbol{A}\|_{L^{2}}}=\frac{\sqrt{\sum_{i=1}^{n}\left(a_{i}-b_{i}\right)^{2}}}{\sqrt{\sum_{i=1}^{n} a_{i}^{2}}},
$$

where $a_{i}$ and $b_{i}$ denote the $i$ th pixel value of observation $\boldsymbol{A}$ and template $\boldsymbol{B}$, respectively, and $\|\cdot\|_{L^{2}}$ is the Euclidean norm. If the template were in perfect agreement with the observation, then $\boldsymbol{B}$ would equal $\boldsymbol{A}$ and thus $F(\boldsymbol{A}, \boldsymbol{B})$ would be zero. If the simulation showed only space $(\boldsymbol{B}=\mathbf{0})$, then $F(\boldsymbol{A}, \boldsymbol{B})$ would be equal to 1 corresponding to a deviation by $100 \%$.

Some images show only a fraction of the primary object. This occurs when the moon enters or leaves the FOV, that is, at the beginning or end of a series. The deviation function $F$ indicates that sufficient confidence for the localization of an object's COF can be achieved if approximately $15 \%$ of the object is visible. Hence, all images containing less than $15 \%$ of the body are not considered for the measurement.

Comparisons of the observations with predicted positions based on current orbit models show, however, in some cases increased scatter for observations along the edge of an image (see Fig. 8b) indicating a higher uncertainty than for observations where the body is fully visible. This agrees with observations by Thomas (1989) who noticed an increase in the uncertainty of a measurement with decreasing visibility of the limb. Data provided with this publication include an indicator of whether the respective observation is based on a full or a partially seen object.

Except for one image series, the secondary objects are completely visible throughout the image sequences. The image sequence of MEX orbit HC146 observed the secondary object only in a few images. Here the images showing only a part of the secondary body are excluded from evaluation.

\subsection{Changes of observation geometry}

The initial assumption that the apparent viewing geometry between camera and close-range object (e.g., Phobos or Deimos) changes only on a minimal scale within each image series is valid for most of the mutual events and the automatic object recognition and evaluation of the data can be performed as described. However, in some cases, the close distance and the resulting viewing geometry change the appearance of the closerange object to a greater extent preventing an evaluation of the entire image series with only one template.

For example, in orbit 12151 the distance between MEX and Phobos is approximately $6500 \mathrm{~km}$. From image 72 to image 89 the distance reduces by about $15 \mathrm{~km}$ which leads to a change of Phobos' apparent diameter in the simulation of more than 1 pixel.

Hence, for such a series one reference image is not sufficient to evaluate all the images of the series with the same quality.
Instead the series is segmented in smaller sequences of images and a new reference template is used for each sequence. As a criterion for a necessary change of the reference image the apparent size of Phobos within the simulated template is evaluated. If it changes by more than 0.16 pixels, then a new reference image is used.

\subsection{Derived quantities}

From the measured apparent positions of the primary and the secondary object their angular distance is computed. For mutual event observations including Jupiter, Saturn, or another far distance object, celestial coordinates for the apparent positions can be derived. Using the measured positions of both objects the difference in right ascension $\triangle \mathrm{RA}$ and declination $\triangle \mathrm{DEC}$ of the primary object with respect to the secondary object (as seen from MEX) is computed and added to the secondary body's predicted position. The far distant object is fixed with respect to the image plane moving only by one percent of a pixel over the time span of one image series. Hence the far distant object can be used as a fiducial point that can help to correct SPICE kernel information on the attitude of the camera with respect to the stellar sky about two axes ( $x$ and $y$ or yaw and pitch). The twist or roll angle, with the rotation axis along the boresight vector of the camera, was reported to be consistent (Pasewaldt et al. 2015; Willner et al. 2008) with the information in the SPICE kernel data and is not separately corrected. Likewise, the scaling factor was reported to be 1.0, with sufficient accuracy throughout the SRC observations (Willner et al. 2008), and is not separately analyzed here.

The derived celestial positions are compared with predictions by current ephemerides models (cf. Sect. 5). In order to also enable such a comparison for images showing Deimos as a secondary object and Phobos as a primary object, the right ascension and declination of Phobos are computed with respect to Deimos. However, Deimos is much closer to MEX than Jupiter or Saturn and thus moving considerably over the time span of each image series. Hence, for these images the obtained right ascension and declination of Phobos are not provided to users via CDS.

\subsection{Accuracy of observations}

In contrast to previous astrometric observations (Oberst et al. 2006; Willner et al. 2008; Pasewaldt et al. 2012, 2015), where the absolute positions of Phobos or Deimos in the stellar sky as seen from the MEX spacecraft were provided, the mutual event observations are free of any influences from spacecraft pointing. This is due to the fact that primarily the angular difference between two observed bodies, that is, the relative position, is observed and provided. Only when Jupiter or Saturn are observed as secondary objects, are the absolute positions of the primary body derived based on predicted positions of Jupiter and Saturn and the observed angular distance to the primary body.

A significantly different than expected spacecraft position with respect to the primary object could result in a simulation of the body from a different perspective than observed - that is, the simulation appears rotated in comparison to the observation. However, MEX' position accuracies have been reported to be of the order of $\pm 500 \mathrm{~m}$ (Oberst et al. 2006; Willner et al. 2008) and more recently even of the order of $\pm(100-200) \mathrm{m}$ (Pischel $\&$ Zegers 2009) resulting in a minor change of the perspective. Of the four observed bodies, Phobos is the object that gets closest to MEX, where the distance between MEX and Phobos is always larger than $6000 \mathrm{~km}$. With a positional error of $500 \mathrm{~m}$ 


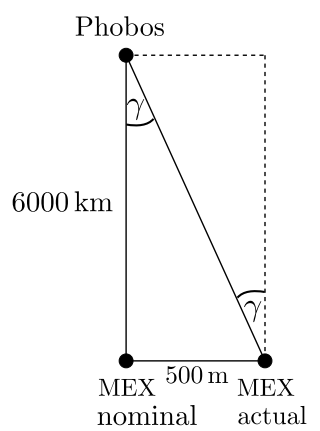

Fig. 6. Translation of MEX' position error $\sigma_{\mathrm{pos}}= \pm 500 \mathrm{~m}$ to angular deviation of the observed object in the image.

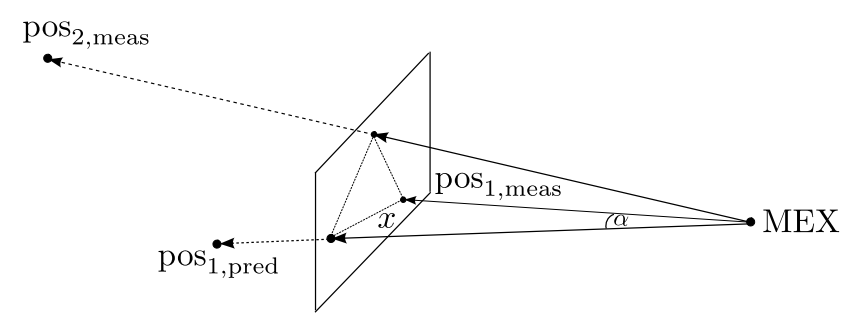

Fig. 7. The perspective projection $x$ of the difference between the primary object's measured ( $\left.\operatorname{pos}_{1, \text { meas }}\right)$ and predicted ( $\left.\operatorname{pos}_{1, \text { pred }}\right)$ position into the object plane.

for MEX, the maximal angular displacement of Phobos in the images amounts to $0.0083 \mathrm{mrad}$, in comparison to an instantaneous FOV for one pixel of 9 mrad (cf. Fig. 6). For Deimos, Jupiter, and Saturn, the potential error due to the spacecraft $(\mathrm{s} / \mathrm{c})$ position error is even smaller. Thus, the s/c position error is considered to be negligible.

Errors may result from the matching between the simulated template and the reference image. Here the remaining difference between the template and the image is critical. In particular for Phobos and Deimos the determination of their respective COF strongly depends on correct shape and rotational models. Errors that are produced by imprecise models can be estimated by means of the standard deviation function, introduced in Sect. 3.2. In the case of Phobos it was observed that the simulated templates, based on the shape model derived by Willner et al. (2014), fitted well to the measurements deviating by only 6 to $11 \%$. In comparison, matching the image series with the derived template the standard deviation $F$ typically reduces to less than $5 \%$.

Simulations of Phobos applying the currently implemented rotational model in the SPICE planetary constants kernel PCK00010.tpc appeared slightly rotated with respect to the observation, most notably for the MEX orbits 9700, 12146, 12151, 15880, and 16346.

This suggested that the rotation model has deficiencies, which has been confirmed by Duxbury (2017, personal comm.) and Stark et al. (2017). Applying the rotational model by Stark et al. (2017), differences between simulation and observations could be reduced for orbits 15880 and 16346 and largely removed in the cases of orbits 9700 and 12151 .

In the case of Deimos there are large deviations between the simulated template and the observations which are most likely caused by the low-resolution shape model that is available. While the rotational model has been improved together with Phobos' rotation model, the global shape model of Deimos will need improvement in the future.
Table 2. Estimated error contributions of the shape model $\left(\sigma_{\text {shape }}\right)$, rotational model $\left(\sigma_{\text {rot }}\right)$ and PSF $\left(\sigma_{\mathrm{PSF}}\right)$ in pixels.

\begin{tabular}{lccc}
\hline \hline Object & $\sigma_{\text {shape }}$ & $\sigma_{\text {rot }}$ & $\sigma_{\text {PSF }}$ \\
\hline Phobos & 0.15 & 0.32 & 0.09 \\
Deimos & 0.68 & 0.06 & 0.04 \\
Jupiter & 0.00 & 0.00 & 0.03 \\
Saturn & 0.00 & 0.00 & 0.06 \\
\hline
\end{tabular}

Imaging errors like blurring, ghost features, and astigmatism described by the SRC's point spread function (PSF, see Oberst et al. 2008, Fig. 1) also constitute a source of error. To best compensate the PSF, the simulation is convolved with the derived PSF (see Sect. 3.1) rather than trying to correct the observations. This leads to a synthetic image most similar to the observation. Hence, it is considered that the PSF is a minor component of the overall error. Imaging errors due to distortions caused by the camera optics are negligible (see Hoffmann et al. 2013).

For each of the observed bodies, the influence of the shape model, rotational parameters, and point spread function was tested. Since the errors due to imperfect shape and rotation models increase with decreasing distance between $\mathrm{s} / \mathrm{c}$ and body, these error components were estimated using images for which the distance between Mars Express and Phobos or Deimos is particularly small (HC151_0085 for Phobos and HC921_0030 for Deimos, where the distances between MEX and the satellite are about $6515 \mathrm{~km}$ and $18330 \mathrm{~km}$, respectively).

For Jupiter and Saturn, errors caused by the shape or rotation model can be ruled out, simply by the large distance to the objects, leaving only the PSF as an error source. The results of the error analysis are provided in Table 2.

Further, differences of the simulated template and the reference image that cannot be eliminated or minimized by a renormalization of the template might also cause errors. For instance, while the simulated templates of Jupiter include the darkening of the limb in comparison to the central area, they do not contain the cloud bands in the equatorial region that can be seen in the measured images. Nevertheless, for Jupiter, the percentage standard deviation for the matching of simulated template and measurement is only about 5 to $6.5 \%$. For Saturn however, the percentage standard deviation for the matching of simulated template and measurement ranges from approximately 14 to $23 \%$ and is thus relatively large.

The total error $\sigma_{1}\left(\sigma_{\mathrm{s}}\right)$ for the line (sample) position of each objects' $\mathrm{COF}$ is computed by

$\sigma_{1 / \mathrm{s}}=\sqrt{\sigma_{\text {shape }}^{2}+\sigma_{\text {rot }}^{2}+\sigma_{\mathrm{PSF}}^{2}+\sigma_{\text {ref, }, 1 / \mathrm{s}}^{2}+\sigma_{\text {fit, }, 1 / \mathrm{s}}^{2}}$,

where $\sigma_{\text {shape }}, \sigma_{\text {rot }}$ and $\sigma_{\mathrm{PSF}}$ denote errors due to imprecision of shape model, rotational parameters, and PSF (cf. Table 2), $\sigma_{\text {ref,l }}$ $\left(\sigma_{\text {ref,s }}\right)$ is the error for the simulated object's line (sample) position in the reference image that is given by the least squares fit for the matching of simulated template and reference image, $\sigma_{\text {fit, } 1}$ $\left(\sigma_{\text {fit,s }}\right)$ is the error for the line (sample) position that is given by the least squares fit for the current image. $\sigma_{\text {shape }}, \sigma_{\text {rot }}$ and $\sigma_{\text {PSF }}$ are applied uniformly throughout the total imaging series while within a series $\sigma_{\text {ref,l }}\left(\sigma_{\text {ref,s }}\right)$ is constant for all images that are analyzed using the same reference image. $\sigma_{\text {fit, }}$ and $\sigma_{\text {fit,s }}$ are determined for each image. 
R. Ziese and K. Willner: Mutual event observations of solar system objects

(a) Deimos-Saturn

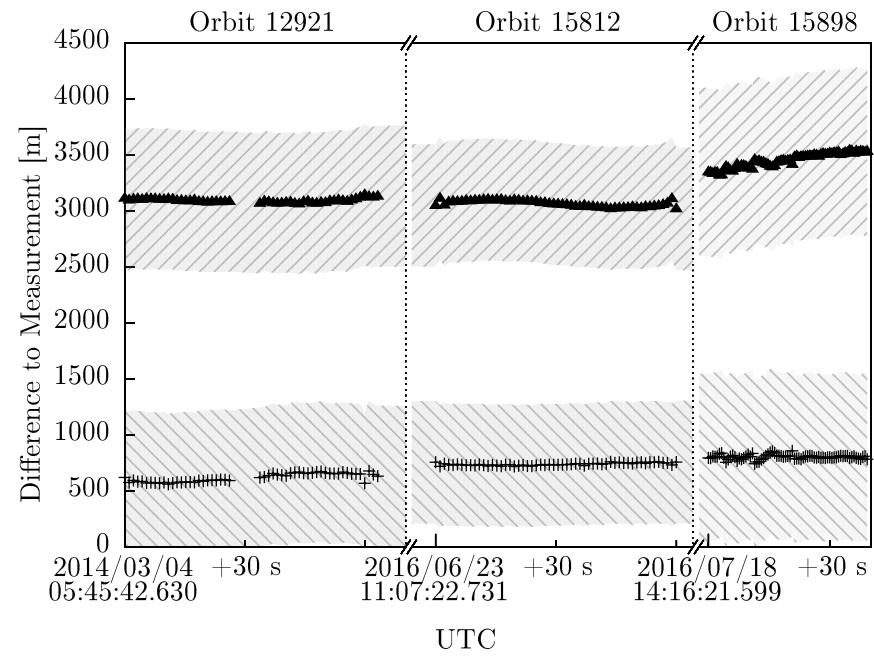

(c) Phobos - Deimos

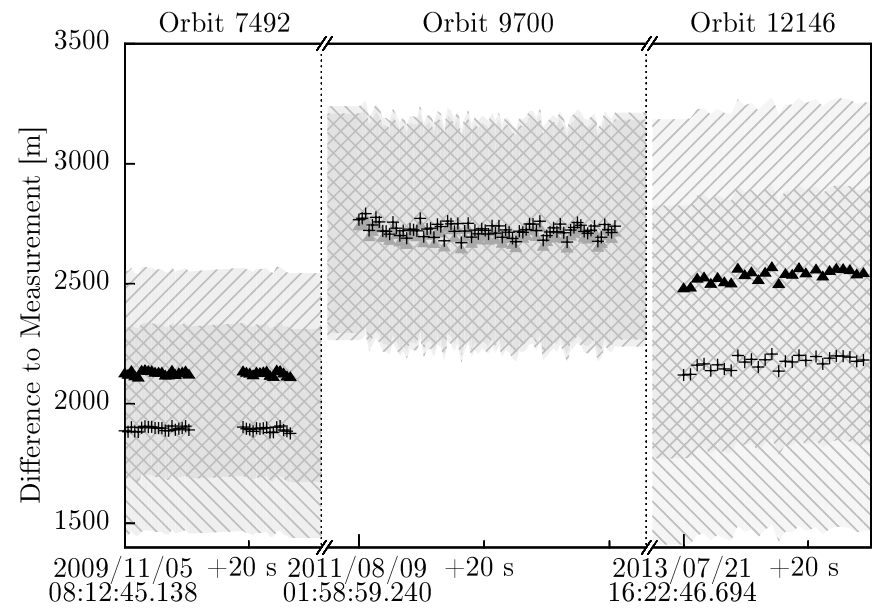

UTC

(e) Phobos - Saturn

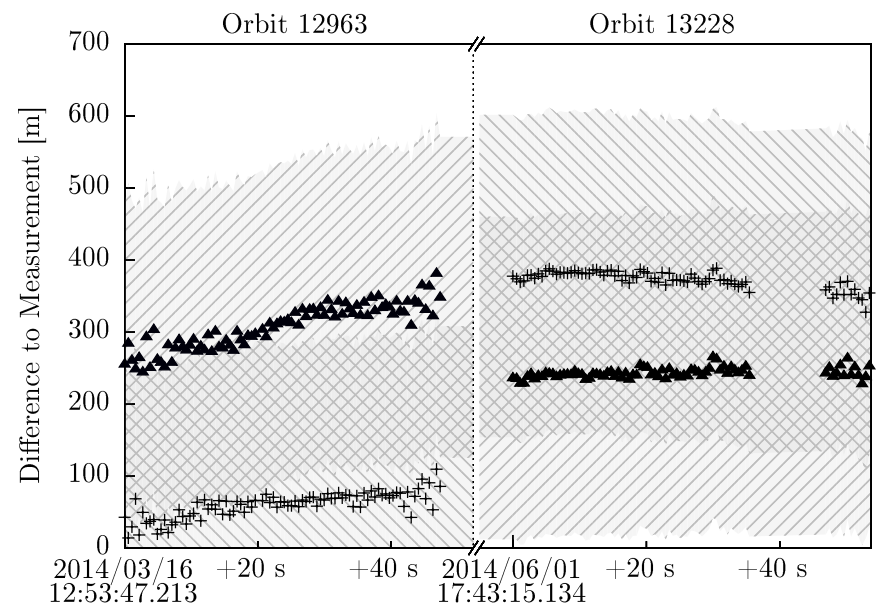

$\mathrm{UTC}$ (b) Phobos-Jupiter

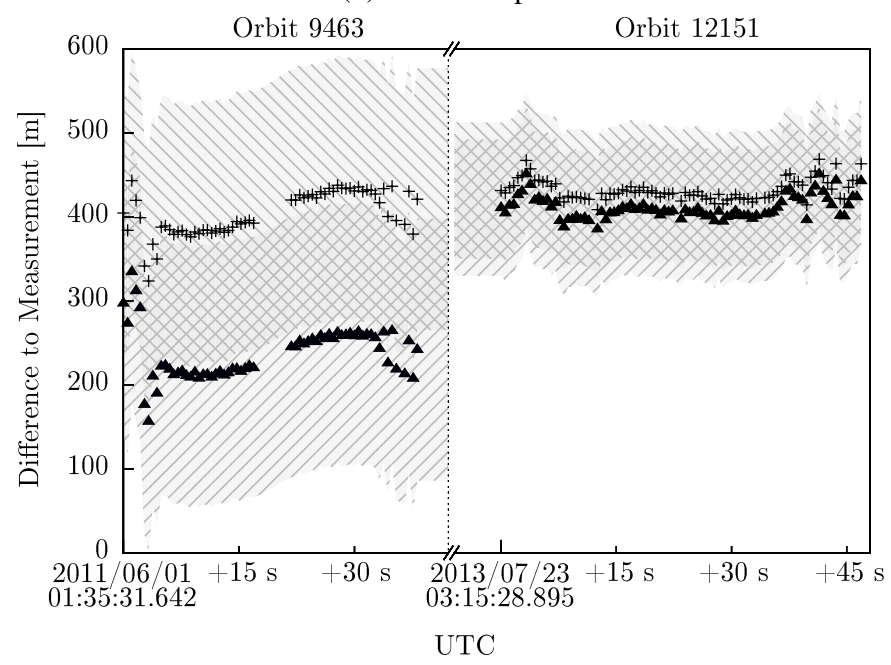

(d) Phobos - Deimos

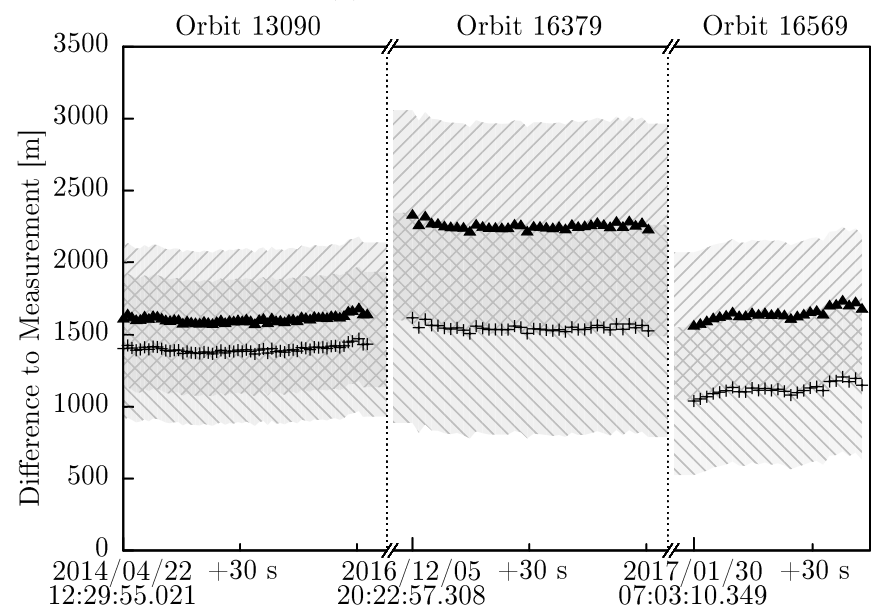

UTC

(f) Phobos - Saturn

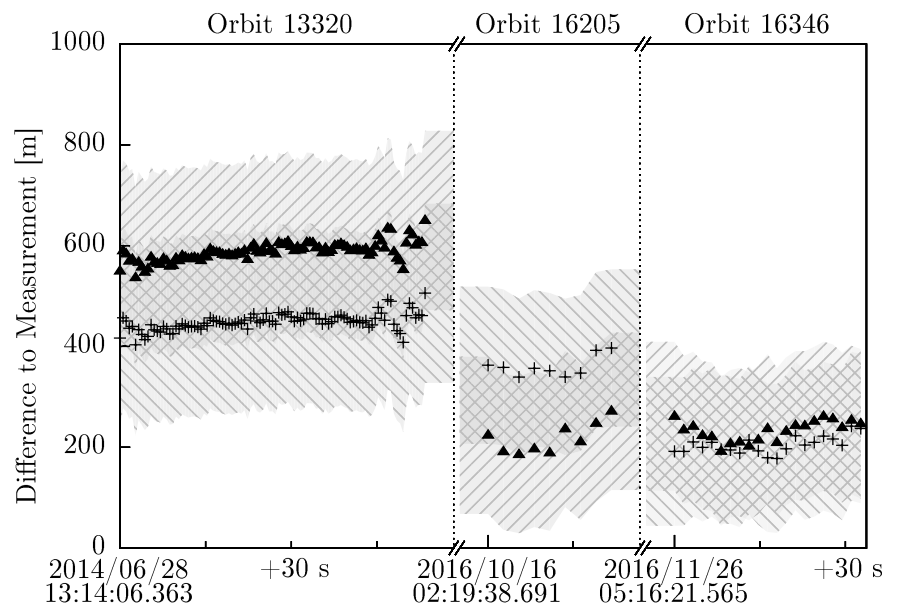

UTC

Fig. 8. Difference of measured and predicted celestial position of the primary object. The depicted values are the perspective projections of the difference vectors into the object plane in meters. The shaded areas indicate the corresponding measurement errors $(3 \sigma)$. 
The errors of the angular separation of the two objects, as well as the right ascension and declination of the primary object, are then derived by linear propagation of uncertainty.

The results are most accurate with increasing accuracy of the shape models of the primary body, or alternatively with increasing distance to the primary body. It is also beneficial when the secondary body is very far away and can be modeled easily. Thus, the most reliable observations are the ones that were deduced from images of Phobos and Jupiter. For these images the error for the angular separation lies between $\pm 0.00063^{\circ}$ and $\pm 0.00089^{\circ}(3 \sigma)$. At the time of these measurements the distance of MEX to Phobos was $6164 \mathrm{~km}$ and $11394 \mathrm{~km}$, respectively. Thus, these errors correspond to a positional error of Phobos with respect to Jupiter, perpendicular to the line of sight, of $\pm(72-176) \mathrm{m}$.

The largest positional errors, $\pm 0.00234^{\circ}(3 \sigma)$ or $\pm 638 \mathrm{~m}$ (at a distance of MEX to Phobos of $15639 \mathrm{~km}$ ), occur for the evaluation of images showing Phobos together with Deimos.

\section{Results}

We have determined the COFs of the planetary bodies that are simultaneously observed in one image and computed their angular separation in 884 images that were acquired between November 2009 and January 2017. The results are provided on CDS in tabular form (see Tables A.1-A.4). For each combination of primary (Phobos or Deimos) and secondary object (Deimos, Jupiter, Saturn) we provide a separate table. Each of the tables contains the image name, the acquisition date and time (image mid-time), the position of Mars Express (w.r.t. to Mars-centered ICRF) at the time of observation, and an indicator of whether the primary body can only be partially seen or is fully visible. Furthermore, the tables provide the measured angular separations between the two observed bodies, as well as the corresponding error $(3 \sigma)$ in degrees and $\mathrm{m}$.

Additionally, if the secondary object is a far distant object, the derived right ascension and declination of the primary object (as seen from MEX) and the predicted right ascension and declination of the secondary object are given together with their errors $(3 \sigma)$.

\section{Comparison with current ephemerides}

In order to relate the presented measurements to current orbit models, the reduced celestial positions of the primary object are compared with the respective values that are predicted by the two most recent ephemerides MAR097 and NOE-4-2015-b, provided by JPL (Jacobson \& Lainey 2014) and the IMCCE, respectively.

Figure 8 shows the differences between measured and predicted celestial positions of the primary object for each image series. These values are the perspective projections $x$ of the difference vectors into the object plane in meters, obtained by means of the nominal distance from MEX to the primary object and the angle $\alpha$ between the directions to the measured and the predicted position; see Fig. 7. The displayed differences only allow for a general comparison but do not indicate any spatial orientation of the difference vector.

However, there are a few occasions when the primary body is moving almost parallel with respect to the image plane and the majority of the difference vector (predicted position minus observed position) is directly visible, such as for the Deimos and Saturn observations in Fig. 8a. The differences shown here range between $560 \mathrm{~m}$ and $858 \mathrm{~m}$ for the NOE-4-2015-b orbit model and $3020 \mathrm{~m}$ and $3543 \mathrm{~m}$ for the MAR097 orbit model when compared against the measurements. The error bars $(3 \sigma)$ displayed show the effect of the observation error on the difference. It becomes clear that the observations are of much higher accuracy in comparison to the current orbit models and can contribute to an improvement of the current knowledge. The ephemerides NOE-4-2015-b are in much better agreement with the Deimos astrometric observations than the orbit model of MAR097. In the case of Phobos observations, however, both orbit models show statistically similar differences with respect to the observations as the observational error is usually larger or only slightly smaller than the variation between the two ephemerides. Nonetheless, the current observations can contribute to an improvement of the orbit models as the error bars indicate a significant deviation of the ephemerides from the observed positions of Phobos.

\section{Summary and conclusions}

In a set of 884 images of mutual events, the angular separation between the two objects was determined. If the secondary object is either Jupiter or Saturn, the right ascension and declination of the primary object with respect to MEX-centered ICRF coordinates could also be derived and are part of the data tables provided with this study. The smallest error on the observations was derived for observations of Phobos, with Jupiter being the secondary body in the image.

Comparisons of the observations with current orbit models, NOE-4-2015-b and MAR097, show an overall better agreement of the NOE-4-2015-b orbit model with the derived observations.

As a next step, SRC images showing Phobos or Deimos and the Earth moon, the Galilean moons, a star, or a star field will be evaluated. Moreover, a few image series also depict Phobos above the Martian surface. This kind of information requires some additional considerations and will also be addressed within a separate publication.

Acknowledgements. The authors thank the HRSC Experiment team at DLR, Institute of Planetary Research, Berlin, and at Freie Universität Berlin, the HRSC Science Team, as well as the Mars Express Project teams at ESTEC, ESOC, and ESAC for their successful planning and acquisition of data as well as for making processed data available to the HRSC team. The valuable discussions with Thomas C. Duxbury, the informative advice on the SRC's optical properties by Klaus-Dieter Matz, and the constructive review comments by Valery Lainey are much appreciated.

\section{References}

Acton, C. H. 2013, in Lunar and Planetary Science Conference, 44, 1224 Bills, B. G., Neumann, G. A., Smith, D. E., \& Zuber, M. T. 2005, J. Geophys. Res., 110, E07004

Borderies, N., \& Yoder, C. F. 1990, A\&A, 233, 235

Christensen, E. J., Born, G. H., Hildebrand, C. E., \& Williams, B. G. 1977, Geophys. Res. Lett., 4, 555

Duxbury, T. C., \& Callahan, J. D. 1988, A\&A, 201, 169

Duxbury, T. C., \& Callahan, J. D. 1989, A\&A, 216, 284

Duxbury, T., Hoffmann, H., Roatsch, T., et al. 2011, Technical Note

Hoffmann, H., Roatsch, T., Elgner, S., \& Matz, K.-D. 2013, SRC Alignment \& Geometric Calibration, NAIF SPICE Kernels, Image Restoration and Vesta, Technical Note

Jacobson, R. A. 2010, AJ, 139, 668

Jacobson, R. A. \& Lainey, V. 2014, Planet. Space Sci. 102, 102, 35

Jaumann, R., Neukum, G., Behnke, T., et al. 2007, Planet. Space Sci., 55, 928

Kolyuka, Y., Tikhonov, V., Ivanov, N., et al. 1991, A\&A, 244, 236

Konopliv, A. S., Yoder, C. F., Standish, E. M., Yuan, D.-N., \& Sjogren, W. L. 2006, Icarus, 182, 23

Lainey, V., Dehant, V., \& Pätzold, M. 2007, A\&A, 465, 1075

Longobardo, A., Palomba, E., Capaccioni, F., et al. 2014, Icarus, 240, 20 
R. Ziese and K. Willner: Mutual event observations of solar system objects

Milne, E. A. 1921, MNRAS, 81, 361

Morley, T. A. 1989, A\&AS, 77, 209

Oberst, J., Matz, K.-D., Roatsch, T., et al. 2006, A\&A, 447, 1145

Oberst, J., Schwarz, G., Behnke, T., et al. 2008, Planet. Space Sci., 56, 473

Pasewaldt, A., Oberst, J., Willner, K., et al. 2012, A\&A, 545, A144

Pasewaldt, A., Oberst, J., Willner, K., et al. 2015, A\&A, 580, A28

Pätzold, M., Andert, T., Jacobson, R., Rosenblatt, P., \& Dehant, V. 2014a, Planet. Space Sci., 102, 86

Pätzold, M., Andert, T. P., Tyler, G. L., et al. 2014b, Icarus, 229, 92

Pischel, R., \& Zegers, T. 2009, Mars Express Science Planning and Operations, ESA SP-1291
Rambaux, N., Castillo-Rogez, J. C., Le Maistre, S., \& Rosenblatt, P. 2012, A\&A, 548, A14

Shkuratov, Y. G., Kreslavsky, M. A., Ovcharenko, A. A., Stankevich, D. G., \& Zubko, E. S. 1999, Icarus, 141, 132

Stark, A., Willner, K., Burmeister, S., \& Oberst, J. 2017, European Planetary Science Congress, EPSC2017-868

Thomas, P. C. 1989, Icarus, 77, 248

Thomas, P. C., Yoder, C. F., Synnott, S. P., et al. 2000, NASA Planetary Data System, 173

Willner, K. 2009, PhD Thesis, Technische Universität Berlin

Willner, K., Oberst, J., Wählisch, M., et al. 2008, A\&A, 488, 361

Willner, K., Shi, X., \& Oberst, J. 2014, Planet. Space Sci., 102, 51 